\title{
Förderpreis Nephrologische Pflege 2013
}

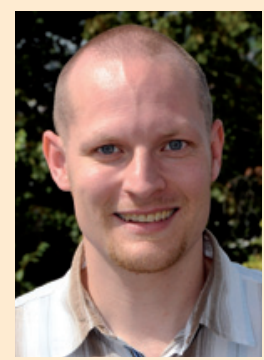

Christian Schäfer, Stuttgart
Nun ist es wieder so weit: In der vorliegenden ersten Ausgabe der Dialyse aktuell im Jahr 2014 können Sie die besten Beiträge von Pflegekräften nachlesen, die sich um den 8. Förderpreis Nephrologische Pflege beworben haben. Wie der Titel des Preises schon sagt, möchte der Georg Thieme Verlag zusammen mit der Arbeitsgemeinschaft für nephrologisches Personal e.V. (AfnP) Menschen fördern, die mit in Manuskriptform gegossenen Arbeiten dazu beitragen, die nephrologische Pflege voranzubringen. Es geht auch darum, Anreize zu setzen, Beiträge und Studien zum Thema nephrologische Pflege zu schreiben, da in diesem Bereich noch immer ein relativ großer Mangel besteht. Eine Motivation für die Einreichung von Beiträgen sind u.a. das Preisgeld von 1500 Euro für den ersten Platz, den 2013 wie gehabt die AfnP und der Thieme Verlag auf dem AfnP-Symposium in Fulda übergeben haben (siehe Artikel ab Seite 8), und die Veröffentlichung der besten Beiträge in der Dialyse aktuell (siehe Artikel ab Seite 28).

Nun zu den Beiträgen selbst: Den ersten Platz und somit den Preis teilten sich diesmal Antje Diedrich und Rebecca Fiedler, die sich als Autorenteam dem Thema „Leitfaden für Dialysepatienten Erstellung und Evaluierung“ annahmen. Hierfür analysierten sie die Wünsche und den Informationsstand von Dialysepatienten mittels eines Fragebogens separat in einer Gemeinschaftspraxis und einer Klinikdialyseabteilung. Basierend auf den Ergebnissen erstellten sie jeweils individuelle Leitfäden. Ein zweiter Fragebogen konnte die positive Wirkung der Leitfäden bestätigen. Dies ist ein wichtiger Beitrag dazu, sicherzustellen, dass ein größtmöglicher Anteil der Informationen, die für Patienten und Angehörige wichtig sind, auch wirklich ankommen und umgesetzt werden können - ein entscheidender Faktor für die Effektivität der Therapie und die Patientensicherheit.

Auf den zweiten Platz kam Carolin Theiß mit ihrem Artikel „Peritonealdialyseambulanz - Aufbau und Strukturierung". Die Peritonealdialyse (PD) ist in Deutschland im Vergleich zur Hämodialyse (HD) wenig verbreitet: Nur ca. $5 \%$ der Dialysepatienten nutzen sie. Prinzipiell sind die beiden Blutreinigungsverfahren aber als ebenbürtig anzusehen, auch wenn sie verschiedene Vor- und Nachteile haben. Umso wichtiger ist es, dass Artikel wie diese aufzeigen, wie der Aufbau und die Etablierung von PD-Ambulanzen erfolgreich geschehen kann. Dadurch lassen sich vielleicht die Leiter anderer Zentren ermutigen, ebenfalls einen Bereich für PD-Patienten einzurichten.

Den dritten Platz belegte Inken Muhs mit ihrer Arbeit „Aktive Bewegung während der Dialysebehandlung - Positive Auswirkungen auf die Patienten“. Bewegung ist die beste „Polypille“ - das wird schnell deutlich, wenn man sich so verschiedene Kategorien wie z. B. die Psyche, die Krebsentstehung und das Herz-Kreislauf-System anschaut: Hier und noch in anderen Bereichen hat körperliche Betätigung eine positive Auswirkung auf den Menschen. Was liegt also näher, als die tägliche Liegezeit der Patienten bei der Dialyse für aktive Bewegung zu nutzen? Die Ergebnisse dieses Vorhabens werden im Beitrag deutlich und sprechen hoffentlich viele andere Dialyseteams an - es wäre zum Wohle der Patienten.

Ich wünsche Ihnen viel Spaß beim Lesen in dieser Ausgabe der Dialyse aktuell! 